\title{
12
}

\section{Stormwater Two-Ramp Drop Structures}

\author{
Subhash C. Jain
}

The chapter summarizes the hydraulic characteristics of the latest type of drop structure, termed two-ramp drop structure, used for diverting flows from nearsurface storm-sewer systems to underground storage tunnels. A series of physical model studies were conducted. This drop structure can be constructed by a tunneling technique that can be more suitable in urban areas where an opencut construction may be expensive and infeasible.

\subsection{Introduction}

Frequent flooding in metropolitan cities around the world along with the enactment of stringent water-quality standards have necessitated the development of improved means of stormwater disposal. Underground storage tunnels are increasingly being used to address the problem. Stormwater or combined sewer overflow (CSO) from near-surface storm-sewer systems is diverted to underground tunnels through a number of vertical drop structures along the tunnels. In this application, a vertical drop structure may be a key component of storm-sewer systems. Considerable work has been done in recent years at the Iowa Institute of Hydraulic Research (IIHR) to improve designs of drop

Jain, S.C. 2000. "Stormwater Two-Ramp Drop Structures." Journal of Water Management Modeling R206-12. doi: 10.14796/JWMM.R206-12.

(C) CHI 2000 www.chijournal.org ISSN: 2292-6062 (Formerly in Applied Modeling of Urban Water Systems. ISBN: 0-9683681-3-1) 
structures which must function effectively and simultaneously as a conveyance structure, an energy dissipator, and a deaeration structure, and yet remain cost effective. (A related paper appears as chapter 12 in monograph 5 in this series).

A typical vertical drop structure, as shown in Figure 12.1, consists of an inlet structure, a vertical drop shaft (with or without a helicoidal ramp), and an adit conduit (with and without a deaeration chamber). The inlet structure controls the flow depth in its approach channel and the flow configuration in the drop shaft. The drop shaft is generally of a constant diameter, for ease of construction, selected to pass the design discharge. The deaeration chamber with an air vent is included to prevent the flow of entrained air into the tunnel. The adit conduit connects the deaeration chamber to the conveyance/detention tunnel.
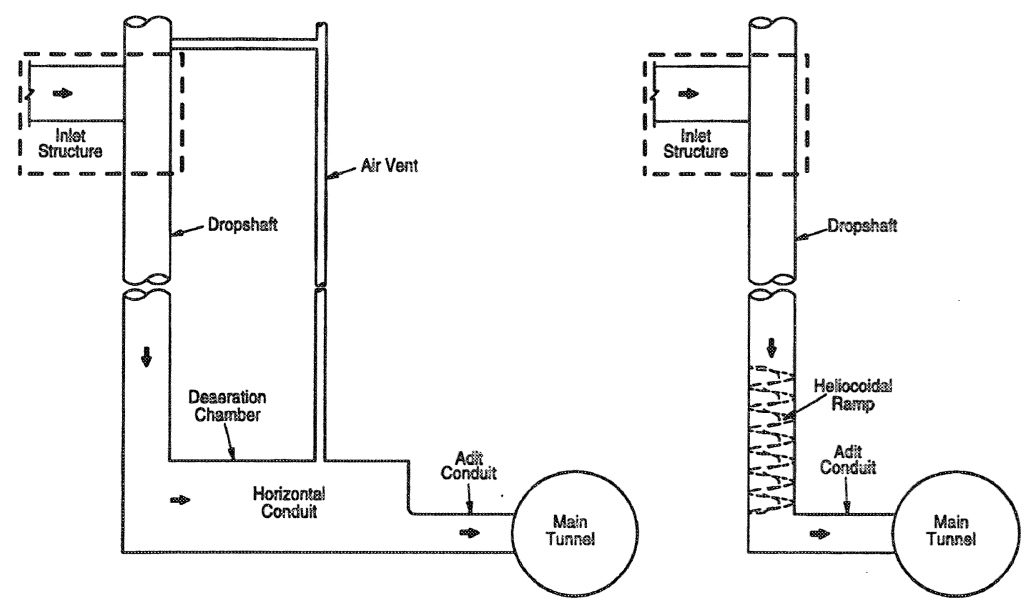

Figure 12.1 Elements of drop structures.

Vortex-flow drop structures with a tangential inlet developed at IIHR, referred to as tangential-inlet drop structures, have been designed for the stormwater-drainage systems in Milwaukee, Wisconsin and Cleveland, Ohio (Jain and Kennedy 1983, 1984; Jain 1984). Lately, a new type of drop structure, referred to as the two-ramp drop structure and shown in Figure 12.2, was developed at IIHR. The deaeration chamber at the bottom of the drop shaft is obviated by providing a helicoidal ramp within the drop shaft. The two-ramp drop structure is an improved version of the helicoidal-ramp drop structures designed for the drainage systems in Phoenix and Tucson, Arizona (Kennedy et al. 1988; Jain and Kennedy 1988). The two-ramp drop structures are under construction in Tokyo, Japan (Jain et al. 1993). The hydraulic characteristics of the two-ramp drop structures were developed by physical model tests and are presented in this chapter. 


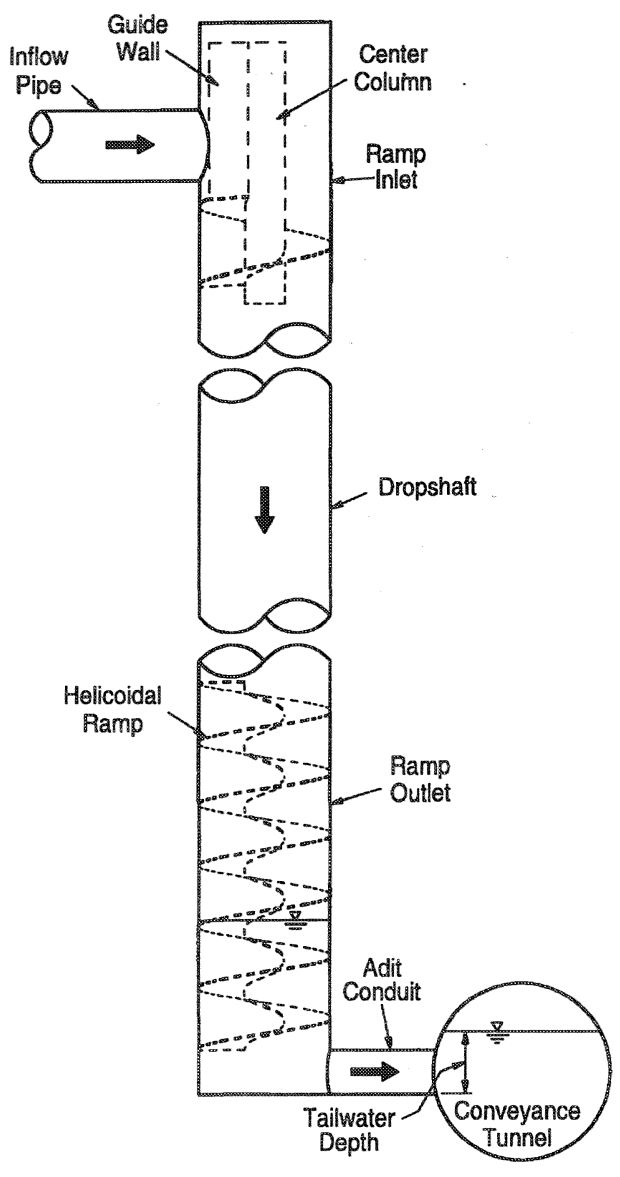

ELEVATION DROPSHAFT

Figure 12.2 Two-ramp drop structure.

\subsection{Geometrical Configuration}

The configuration of the two-ramp drop structure is shown in Figure 12.2. It has three main elements: a ramp inlet; a vertical drop shaft; and a ramp outlet. The details of the ramp inlet are presented in Figure 12.3. The inlet consists of an inflow pipe and a one-revolution helicoidal ramp in the drop shaft, referred to as the inlet ramp. The inflow pipe is connected radially to the drop shaft. The inlet ramp has a central circular hollow column and a guide wall at its upstream end. The drop shaft is vertical and has a constant diameter. The ramp outlet consists of a six-revolution helicoidal ramp and an adit conduit, referred to as the outlet ramp. The adit conduit is connected radially to the drop shaft. 


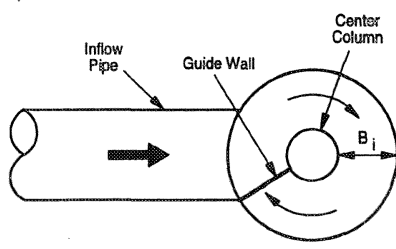

PLAN VIEW OF DROPSHAFT INLET

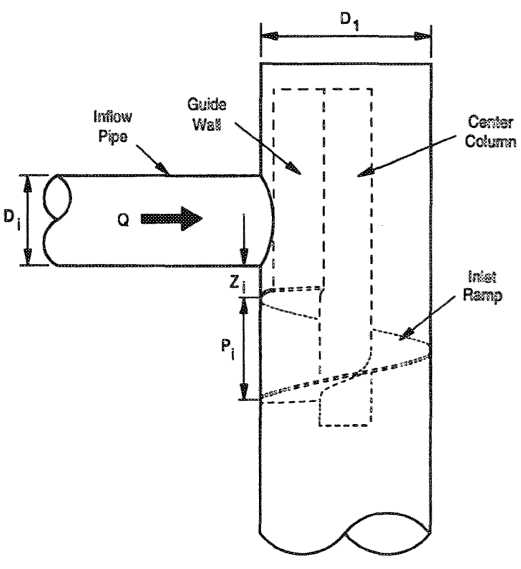

ELEVATION OF DROPSHAFT INLET

Figure 12.3 Details of the inlet ramp.

\subsection{Flow Configuration}

The flow configuration in the drop structure observed in the model tests is presented in this section. The flow from the inflow pipe enters the drop shaft radially, wherein it is deflected by the guide wall and the central column and swirls down the inlet ramp. At low discharges, the inlet helical channel is partly full; the water in the helical channel runs along the drop-shaft wall as a free-surface flow. A small amount of air is entrained by the flow as it hits the inlet ramp and the central column; the entrained air is released through the free surface over the ramp. With increasing water discharge, the flow depth in the inflow pipe increases, the inlet helical channel runs more full, and the flow entrains more air due to the formation of a strong vortex at the helical-channel entrance. At a certain discharge, the inlet helical channel runs full with air-water mixture. With further increase in discharge the inflow pipe runs full and the air entrainment goes down. Eventually the inlet helical channel runs full without air.

The flow leaves the inlet ramp as a swirling jet and remains attached to the drop-shaft wall. As the water swirls down the drop shaft, the flow becomes increasingly vertical as a result of the increase in the vertical velocity due to the 
gravity force and the decrease in the tangential velocity due to the friction force. At the same time the jet becomes increasingly thinner. The jet in a long drop shaft eventually becomes vertical and attains a limiting thickness and velocity.

The swirling, wall-attached flow is intercepted by the top revolution of the outlet ramp. A large amount of air is entrained by the flow as it is intercepted by the ramp. Due to an inward pressure gradient produced by the swirl, the entrained air converges towards the center of the drop shaft where it is subsequently released into the central air core within about two to three revolutions of the ramp. The flow without air swirls down the ramp until it plunges in the pool formed at the bottom of the drop shaft, and once again entrains air during plunging. A significant amount of air is released back into the central air core, and the remaining air is transported into the main tunnel through the adit conduit. The amount of air transport decreases with increasing pool depth, which in turn increases with increasing tailwater depth and water discharge.

\subsection{Hydraulic Analysis}

The hydraulic performance of the drop structure is primarily controlled by its inlet (inflow pipe and inlet ramp) and outlet (outlet ramp and adit conduit). The inlet ramp generates the swirling motion in the drop shaft and controls the flow depth in the inflow pipe. The outlet ramp regenerates the swirling motion which forces the entrained air into the central air core. The adit conduit controls the pool depth at the bottom of the drop shaft, which in turn affects the air transport into the storage tunnel through the adit conduit.

\subsubsection{Head-Discharge Relation}

The head-discharge relation in a ramp inlet (see Figure 12.4) is controlled by the inflow- pipe diameter, $D_{i}$; the drop-shaft diameter, $D_{1}$; the helicoidal-ramp pitch, $\mathrm{P}_{\mathrm{i}}$; the helicoidal-ramp width, $\mathrm{B}_{\mathrm{i}}$ and the distance, $\mathrm{Z}_{\mathrm{i}}$, between the invert of the inflow pipe and the top of the inlet ramp. A typical head-discharge curve for the drop structure is shown in Figure 12.4, where $\mathrm{H}=$ piezometric head in the inflow pipe relative to the invert of the inflow pipe and $Q=$ water discharge in the inflow channel. The curve is characterized by three flow regions: a critical-flow, a transition-flow, and a pipe-flow region. In the critical-flow region $(\mathrm{OA})$, the flow depth in the inflow pipe is close to the critical depth, because the flow behavior at the downstream end of the inflow pipe is similar to that of a free overfall. Though the flow depth in the critical-flow region does not depend on the inletramp configuration, the extent of the region (location of point $\mathrm{A}$ ) does. The flow depth $\mathrm{H}_{\mathrm{A}}$ at the end of the critical-depth region is less than the inflow-pipe diameter; the inflow pipe is not full. The discharge at flow depth $\mathrm{H}_{\mathrm{A}}$ is represented 


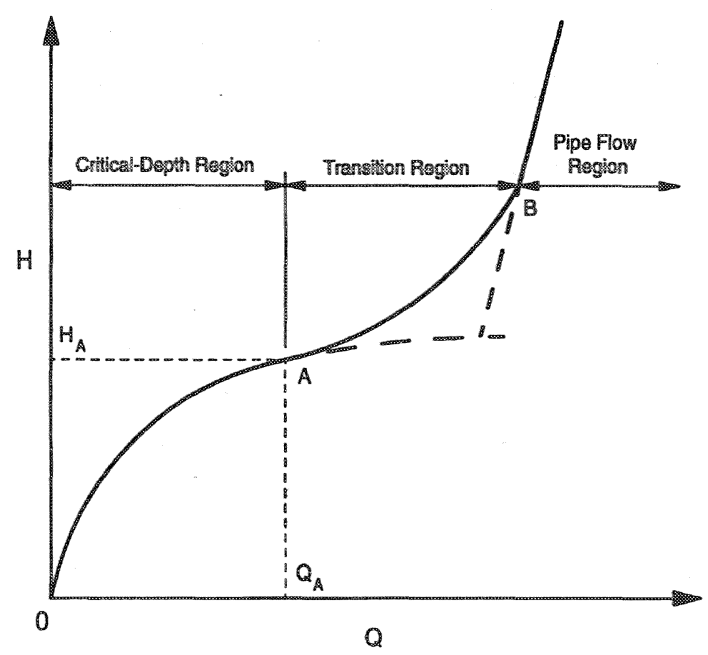

Figure 12.4 Head-discharge curve.

by $Q_{A}$. For discharges higher than $Q_{A}$, the flow depth in the inflow pipe is affected by the flow conditions in the inlet ramp. At a certain discharge $Q>Q_{A}$, the inflow pipe runs full and the inlet helical channel carries the air-water mixture due to air entrainment by the free-surface vortices near the entrance to the inlet ramp. The air entrainment decreases with increasing discharge and ceases at discharge $Q_{B}$. The region $A B$ is the transition-flow region. At discharges higher than $Q_{B}$, both the inflow pipe and the inlet helical channel run full like a pipe flow; this flow region is termed the pipe-flow region.

The inflow pipes in most storm-drainage systems are designed to handle the maximum flow without surcharge. The flow in a partly full inflow pipe is primarily controlled by the inflow-pipe diameter, $\mathrm{D}_{\mathrm{i}}$ and gravitational acceleration, $g$. The head-discharge relation for the critical-flow region is given by (Ali 1993):

$$
\frac{H}{D_{i}}=C\left(\frac{Q^{2}}{g D_{i}^{5}}\right)^{1 / 4}
$$

where:

$$
\begin{aligned}
& C=1.2 \text { for } 2.86>D_{1} / D_{i}>1.92 \text {, and } \\
& C=1.0 \text { for } D_{1} / D_{i}=1.12 .
\end{aligned}
$$

The upper limit of the critical-flow region (location of point A in Figure 12.4) depends on the discharge, the inflow-pipe diameter, and the inlet-ramp configuration. The limit increases with increasing helical-channel area and the distance $\mathrm{Z}_{\mathrm{i}}$ between the invert of the inflow pipe and the top of the inlet ramp. An expression for the limit is not available due to lack of sufficient experimental data. 


\subsubsection{Flow in Drop Shaft}

The drop shaft is a constant diameter, vertical circular pipe that can be divided in three segments. The upper segment is a part of the inlet and has a length (between the invert of the inflow pipe and the bottom of the inlet ramp) of about $1.50 D_{1}$ for $D_{1} / D_{i}=1.12$ and about $0.79 D_{1}$ for $D_{1} / D_{i}=1.92$. The bottom segment is a part of the outlet and has a length (between the top of the outlet ramp and the invert of the adit conduit, described in the next section) of about $3.25 \mathrm{D}_{1}$. The middle segment, whose length depends on the elevation difference between the crown of the inflow pipe and the invert of the outflow pipe, has no helicoidal ramp. For an elevation difference of less than about $4 D_{1}$ to $5 D_{1}$, there is no middle segment of the drop shaft; the drop shaft all along its length contains a continuous ramp consisting of the inlet and outlet ramps.

The angular motion imparted to the flow by the inlet ramp forces the water to cling to, and flow down, the drop-shaft wall in the form of an annular swirling jet. The swirling jet gradually loses its angular velocity due to wall shear and gains its vertical velocity due to gravitational acceleration. In the case of a very long drop shaft the annular jet eventually becomes vertical and attains its limiting fall velocity similar to that in the tangential-inlet drop structure (Jain and Kennedy1983).

\subsubsection{Flow in Ramp Outlet}

The ramp outlet is designed so that the air-transport rate to the storage tunnel is less than a specified value. A typical ramp outlet is shown in Figure 12.5. It consists of a six-revolution helicoidal ramp. The geometry of the outlet is characterized by the diameter of the adit conduit, $\mathrm{D}_{3}$; the drop-shaft diameter, $\mathrm{D}_{1}$; the helicoidal-ramp pitch, $\mathrm{P}_{0}$; and the helicoidal-ramp width, $\mathrm{B}_{0}$.

The swirling, wall-attached flow along the drop shaft is intercepted by the outlet ramp. The discharge along the top revolution of the ramp increases with increasing interception of the flow. A large amount of air is entrained as the flow is intercepted by the ramp. As the air-water mixture moves down the ramp, the entrained air converges, due to radial pressure gradient, towards the center of the drop shaft where it is released into the central air core within the upper three revolution of the ramp. Thereafter the flow in the helical channel is without air until it plunges in the pool formed at the bottom of the drop shaft. Once again air is entrained during plunging, the air bubbles converge towards the center of the drop shaft, and a significant amount of entrained air is released into the central air core. The remaining entrained air is conveyed into the storage tunnel through the adit conduit. The rate of air transport into the tunnel decreases with increasing pool depth in the drop shaft. The pool depth in the drop shaft increases with increasing water discharge and water level in the conveyance or storage tunnel, and decreasing diameter of the adit conduit. 


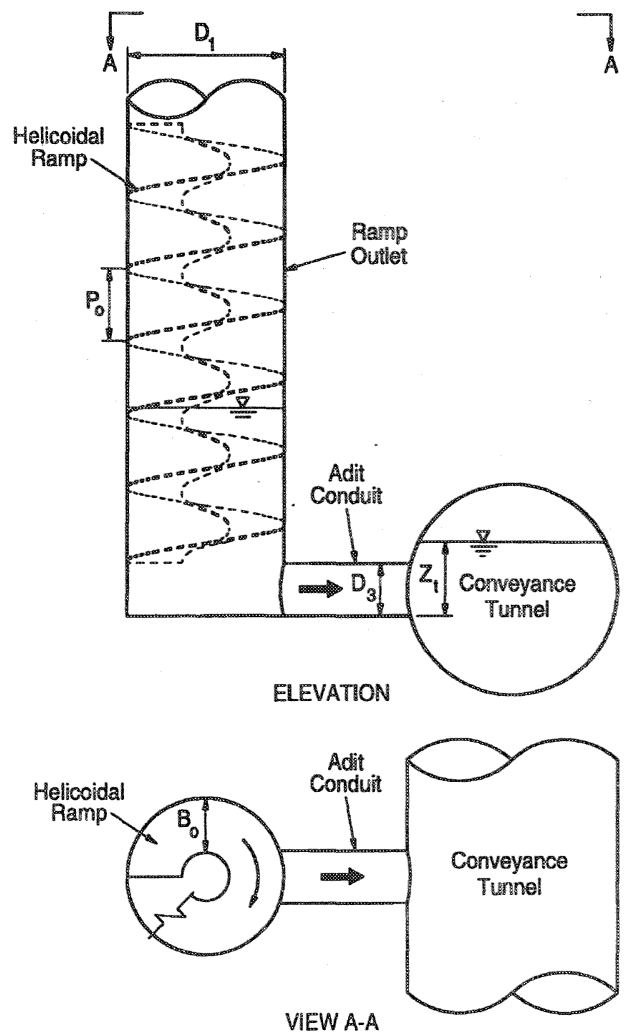

Figure 12.5 Details of the outlet ramp.

The outlet ramp should be able to handle the design discharge. The flow capacity of a ramp, which depends on the pitch and the width of the ramp, can be determined from the Darcy-Weisbach equation as shown below. The number of revolutions required for the outlet ramp depends on the extents of the two airentrainment regions in the ramp; the two regions should not interact with each other to minimize the air transport into the adit conduit. The upper region extends about three revolutions. The lower region depends on the pool depth that in turn depends on discharge and tailwater depth. The extent of the lower region in most cases is less than about three revolutions. Hence the outlet ramp consists of about six revolutions.

The continuity and the Darcy-Weisbach equations are used to relate the discharge to the ramp geometry and the relations are given by:

$$
Q=V w d
$$




$$
V=\left(\frac{8 g R S}{f}\right)^{1 / 2}
$$

in which:

$$
\begin{aligned}
\mathrm{Q} & =\text { discharge } \\
\mathrm{V} & =\text { mean flow velocity } \\
\mathrm{d} & =\text { mean flow depth } \\
\mathrm{w} & =\text { width of the helical channel } \\
\mathrm{S} & =\text { average streamwise slope } \\
\mathrm{R} & =\text { hydraulic radius, and } \\
\mathrm{f} & =\text { Darcy-Weisbach friction factor. }
\end{aligned}
$$

These parameters are related to the ramp geometry (Kennedy et al. 1988).

The friction factors for helical-channel flows are much higher than those for equivalent straight-channel flows. The increase of friction factor for swirling flows (Kennedy et al. 1988) is due to (i) the intensification of turbulence by the concave boundary; (ii) the development of Goertler vortices along the drop-shaft wall; and (iii) the generation of a pair of secondary-flow vortices. The friction factor can be assumed to be the sum of two components, i.e. $f=f_{c}+f_{s}$, where $f_{c}=$ friction factor due to curvature; and $f_{s}=$ friction factor for an equivalent straight channel.

It is judged that the secondary-flow vortices are the principal source for the increase in friction factor and are primarily controlled by the flow geometry. The increase in friction factor due to curvature, $f$, , should be a function of $d / D_{1}$ and $w / d$ (Hayden 1993). The other component of the friction factor, $f_{s}$, can be determined either for a given Reynolds number and relative roughness from the Moody diagram, or for a given Manning roughness coefficient, $n$, from the following equation

$$
f_{S}=\frac{8 g n^{2}}{R^{1 / 3}}
$$

Equation 12.4 is obtained from Darcy-Weisbach and Manning equations, and is valid for fully rough flows for which friction factor is independent of the Reynolds number. The flows in the prototype are expected to be in the fully rough region.

\subsubsection{Air Transport}

On using dimensional analysis, the concentration of air transported to the adit conduit can be expressed as:

$$
C=f\left(D_{3^{*}}, Z_{t^{*}}, F_{r}, R_{e}\right)
$$


in which:

where:

$$
\begin{aligned}
C= & \text { air concentration defined as ratio of the volume flux of } \\
& \text { air to the volume flux of water } \\
D_{3^{*}}= & D_{3} / D_{1}=\text { normalized outflow-pipe diameter; } \\
Z_{t^{*}}= & Z_{\downarrow} / D_{3}=\text { normalized tailwater depth, }
\end{aligned}
$$

$Z_{t}=$ elevation difference between tailwater level and invert level of the outflow pipe

$\mathrm{F}_{\mathrm{r}}=\mathrm{Q} /\left(\mathrm{gD}_{3}^{5}\right)^{1 / 2}=$ Froude number, and

$\mathbb{R}_{\mathrm{e}}=\mathrm{Q} /\left(\mathrm{D}_{3} v\right)=$ Reynolds number, where $v=$ kinematic viscosity of water. The effect of Reynolds number can be neglected as the flow in the outlet is fully turbulent.

A typical variation of air concentration with Froude number for a fixed normalized tailwater depth and outflow-pipe diameter shows that the air concentration first increases, reaches a maximum value, and then decreases with increasing Froude number. Such behavior of air concentration is due to counteracting effects of the two flow parameters, pool depth and outflow velocity. Air concentration is expected to increase with decreasing pool depth and increasing outflow velocity. But both pool depth and outflow velocity are expected to increase with increasing Froude number, which in turn increases with increasing water discharge and decreasing adit-conduit diameter.

The similarity relations for air concentration (Hayden 1993) can be used to determine the rate of air transport to the main tunnel. Figure 12.6 presents the normalized air concentration as a function of normalized Froude number; air concentration is normalized by the peak air concentration, $\mathrm{C}_{\mathrm{p}}$, and Froude number is normalized by the Froude number at the peak air concentration, $F_{\mathrm{rp}}$. It should be mentioned that air-entrainment and transport rates are underestimated in small-scale models.

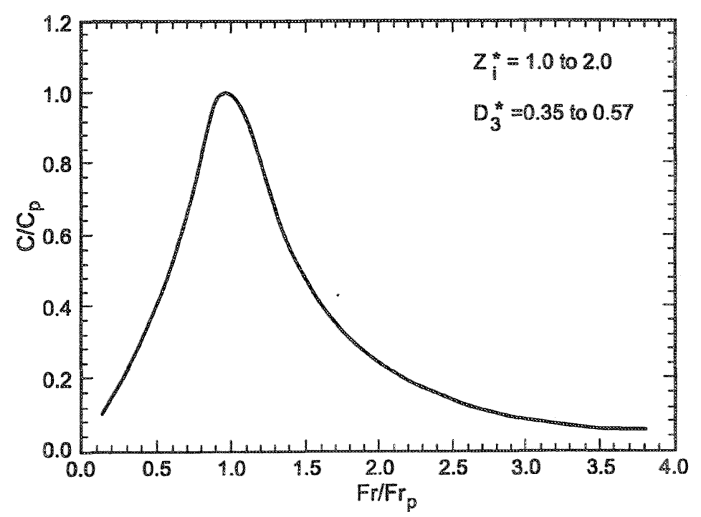

Figure 12.6 Normalized concentration curve. 


\subsection{Conclusions}

This investigation demonstrated the feasibility and practicability of two-ramp drop structures that can be constructed by a tunneling technique. The friction factor is significantly higher for helical-channel flows than for equivalent straight-channel flows. The concentration of air transported out of the drop shaft is very small.

\section{References}

Ali, M.A.M. (1993) "Hydraulic performance of helicoidal ramp radial inlets," M.S. Thesis, Civil and Environmental Engineering, The University of Iowa, Iowa City, Iowa.

Hayden, W.S. (1993) "Hydraulic performance of a radial outlet of a double-ramp drop structure," M.S. Thesis, Civil and Environmental Engineering, The University of Iowa, Iowa City, Iowa.

Jain, S.C. (1984) "Tangential vortex-inlet," Journal of Hydraulic Engineering, ASCE, Vol. 110, No. 12, December.

Jain, S.C., Hayden, W.S., and Ali, M.A.M. (1993) "Novel truncated-ramp drop structures," IIHR Limited Distribution Report No. 206, Iowa Institute of Hydraulic Research, The University of Iowa, Iowa City, Iowa, February.

Jain, S.C. and Kennedy, J.F. (1983) "Vortex-flow dropstructures for the Milwaukee Metropolitan Sewerage District inline storage system," IIHR Report No. 264, Iowa Institute of Hydraulic Research, The University of Iowa, Iowa City, Iowa.

Jain, S.C. and Kennedy, J.F. (1984) "Hydraulic design of SWI drop structures, City of Cleveland," IIHR Limited Distribution Report No. 110, Iowa Institute of Hydraulic Research, The University of Iowa, Iowa City, Iowa.

Jain, S.C. and Kennedy, J.F. (1988) "Model study of Price Road tunnel helicoidal-ramp drop structures," IIHR Limited Distribution Report No. 152, Iowa Institute of Hydraulic Research, The University of Iowa, Iowa City, Iowa.

Kennedy, J.F., Jain, S.C., and Quinnones, R. (1988) "The helicoidal-ramp dropshaft," Journal of Hydraulic Engineering, ASCE, Vol. 114, No. 3, March. 


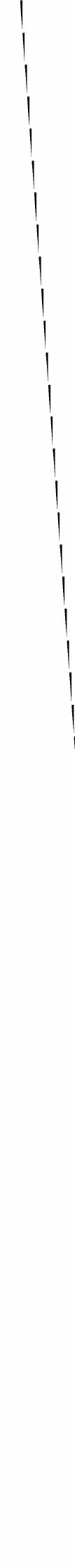

